\title{
Center of mass energy and system-size dependence of photon production at forward rapidity at $\mathrm{RHIC}$
}

\author{
B. I. Abelev ${ }^{\text {h }}$ M. M. Aggarwal ${ }^{\text {ad }}$, Z. Ahammed ${ }^{\text {au, }}$, \\ A. V. Alakhverdyants ${ }^{\mathrm{q}}$, B. D. Anderson ${ }^{\mathrm{r}}$, D. Arkhipkin ${ }^{\mathrm{c}}$, \\ G. S. Averichev ${ }^{\mathrm{q}}$, S. K. Badyal ${ }^{\mathrm{p}}$, J. Balewski ${ }^{\mathrm{v}}$, \\ O. Barannikova $^{\text {h }}$, L. S. Barnby ${ }^{\text {b }}$, J. Baudot ${ }^{\circ}$, S. Baumgart ${ }^{\text {ba }}$, \\ D. R. Beavis ${ }^{\mathrm{c}}, \mathrm{R}$. Bellwied ${ }^{\text {ay }}, \mathrm{F}$. Benedosso ${ }^{\text {aa }}$, \\ M. J. Betancourt ${ }^{\mathrm{v}}$, R. R. Betts ${ }^{\mathrm{h}}$, A. Bhasin ${ }^{\mathrm{p}}$, A. K. Bhati ${ }^{\text {ad }}$, \\ H. Bichsel ${ }^{\text {aw }}$, J. Bielcik ${ }^{j}$, J. Bielcikova ${ }^{k}$, B. Biritz ${ }^{f}$, \\ L. C. Bland ${ }^{\text {c }}$, I. Bnzarov ${ }^{q}$, M. Bombara ${ }^{\text {b }}$, B. E. Bonner ${ }^{\text {aj, }}$, \\ J. Bouchet $^{\mathrm{r}}$, E. Braidot ${ }^{\text {aa }}$, A. V. Brandin ${ }^{\mathrm{y}}$, E. Bruna ${ }^{\mathrm{ba}}$, \\ S. Bueltmann ${ }^{\text {ac }}$, T. P. Burton ${ }^{\text {b }}$ M. Bystersky ${ }^{\text {k }}$, X. Z. Cai ${ }^{\text {an }}$, \\ H. Caines ${ }^{\text {ba }}$, M. Calderón ${ }^{\mathrm{e}}$, O. Catu ${ }^{\text {ba }}$, D. Cebra ${ }^{\mathrm{e}}$, \\ R. Cendejas ${ }^{f}$, M. C. Cervantes ${ }^{\text {ap }}$, Z. Chajecki ${ }^{\text {ab }}$, \\ P. Chaloupka ${ }^{k}$, S. Chattopadhyay ${ }^{\text {au }}$, H. F. Chen ${ }^{\text {a }}$, \\ J. H. Chen ${ }^{\mathrm{r}}$, J. Y. Chen ${ }^{\mathrm{az}}$, J. Cheng ${ }^{\text {ar }}$, M. Cherney ${ }^{\mathrm{i}}$, \\ A. Chikanian ${ }^{\text {ba }}$, K. E. Choi ${ }^{\text {ah }}$, W. Christie $^{\text {c }}$, R. F. Clarke ${ }^{\text {ap }}$, \\ M. J. M. Codrington ${ }^{\text {ap }}$, R. Corliss ${ }^{\text {v }}$, T. M. Cormier ay, \\ M. R. Cosentino ak, J. G. Cramer ${ }^{\text {aw }}$, H. J. Crawford ${ }^{d}$, D. Das ${ }^{\mathrm{e}}$, \\ S. Das ${ }^{\text {au }}$, S. Dash ${ }^{\ell}$, M. Daugherity ${ }^{\text {aq }}$, L. C. De Silva ${ }^{\text {ay }}$, \\ T.G. Dedovich ${ }^{\mathrm{q}}$, M. DePhillips ${ }^{\mathrm{c}}$, A. A. Derevschikov ${ }^{\text {af }}$, \\ R. Derradi de Souza ${ }^{g}$, L. Didenko ${ }^{c}$, P. Djawotho ${ }^{\text {ap }}$, \\ S. M. Dogra ${ }^{\mathrm{p}}$, X. Dong ${ }^{\mathrm{u}}$, J. L. Drachenberg ${ }^{\mathrm{ap}}$, J. E. Draper ${ }^{\mathrm{e}}$, \\ J. C. Dunlop ${ }^{c}$, M. R. Dutta Mazumdar au, L. G. Efimov ${ }^{\mathrm{q}}$, \\ E. Elhalhuli ${ }^{\text {b }}$, M. Elnimr ay , J. Engelage ${ }^{\text {d }}$, G. Eppley ${ }^{\text {aj, }}$, \\ B. Erazmus ${ }^{\text {ao }}$, M. Estienne ${ }^{\text {ao }}$, L. Eun ${ }^{\text {ae }}$, P. Fachini ${ }^{\text {c }}$, \\ R. Fatemi ${ }^{\mathrm{s}}$, J. Fedorisin ${ }^{\mathrm{q}}$, A. Feng ${ }^{\text {az }}$, P. Filip ${ }^{\mathrm{q}}$, E. Finch ${ }^{\text {ba }}$, \\ V. Fine ${ }^{\text {c }}$, Y. Fisyak ${ }^{\text {c }}$, C. A. Gagliardi ${ }^{\text {ap }}$, L. Gaillard ${ }^{\text {b }}$, \\ D. R. Gangadharan ${ }^{f}$, M. S. Ganti au, E.J. Garcia-Solis ${ }^{\text {h }}$,
} A. Geromitsos ${ }^{\text {ao }}$, F. Geurts ${ }^{\text {aj }}$, V. Ghazikhanian ${ }^{\text {f }}$, P. Ghosh ${ }^{\text {au }}$, Y. N. Gorbunov ${ }^{i}$, A. Gordon ${ }^{\text {c }}$, O. Grebenyuk ${ }^{u}$, D. Grosnick ${ }^{\text {at }}$, 
B. Grube ${ }^{\text {ah }}$, S.M. Guertin ${ }^{\text {f }}$, K. S. F. F. Guimaraes ${ }^{\text {ak }}$,

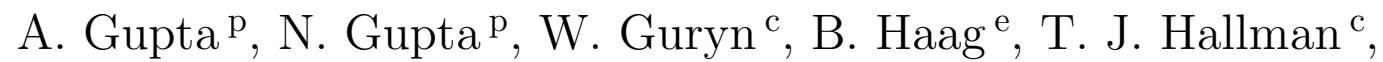
A. Hamed ap, J. W. Harris ${ }^{\text {ba }}$, W. He ${ }^{\text {n }}$, M. Heinz ${ }^{\text {ba }}$, S. Hepplemann ${ }^{\text {ae }}$, B. Hippolyte ${ }^{\circ}$, A. Hirsch ${ }^{\text {ag }}$, E. Hjort ${ }^{\text {u, }}$ A. M. Hoffman ${ }^{\text {v }, ~ G . ~ W . ~ H o f f m a n n ~}{ }^{\text {aq }}$, D. J. Hofman ${ }^{\text {h}}$, R. S. Hollis ${ }^{\text {h}}$, H. Z. Huang ${ }^{\text {f }}$, T. J. Humanic ${ }^{\text {ab }}$, L. Huo ${ }^{\text {ap }}$, G. Igo ${ }^{\mathrm{f}}$, A. Iordanova ${ }^{\mathrm{h}}$, P. Jacobs ${ }^{\mathrm{u}}$, W. W. Jacobs ${ }^{\mathrm{n}}$, P. Jakl ${ }^{\mathrm{k}}$, C. Jena ${ }^{\ell}$, F. Jin ${ }^{\text {an }}$, C. L. Jones ${ }^{\mathrm{v}}$, P. G. Jones ${ }^{\mathrm{b}}$, J. Joseph ${ }^{\mathrm{r}}$, E. G. Judd ${ }^{\text {d, S. Kabana }}{ }^{\text {ao }}$, K. Kajimoto aq, K. Kang ar, J. Kapitan ${ }^{\mathrm{k}}$, K. Kauder ${ }^{\text {h}}$, D. Keane ${ }^{\mathrm{r}}$, A. Kechechyan ${ }^{\mathrm{q}}$, D. Kettler ${ }^{\text {aw }}$, V. Yu. Khodyrev ${ }^{\text {af }}$, D. P. Kikola ${ }^{u}$, J. Kiryluk ${ }^{\text {u }}$, A. Kisiel ${ }^{\text {av }}$, S. R. Klein ${ }^{\text {u }}$, A. G. Knospe ${ }^{\text {ba }}$, A. Kocoloski ${ }^{\text {}}$, D. D. Koetke ${ }^{\text {at }}$, J. Konzer ${ }^{\text {ag }}$, M. Kopytine ${ }^{\mathrm{r}}$, I. Koralt ${ }^{\mathrm{ac}}$, W. Korsch ${ }^{\mathrm{s}}$, L. Kotchenda ${ }^{\mathrm{y}}$, V. Kouchpil ${ }^{\mathrm{k}}$, P. Kravtsov ${ }^{\mathrm{y}}$, V. I. Kravtsov ${ }^{\text {af }}$, K. Krueger ${ }^{a}$, M. Krus ${ }^{j}$, C. Kuhn ${ }^{\circ}$, L. Kumar ad, P. Kurnadi ${ }^{\mathrm{f}}$, M. A. C. Lamont ${ }^{\mathrm{c}}$, J. M. Landgraf ${ }^{\mathrm{c}}$,

S. LaPointe ${ }^{\text {ay }}$, J. Lauret ${ }^{\mathrm{c}}$, A. Lebedev ${ }^{\mathrm{c}}$, R. Lednicky ${ }^{\mathrm{q}}$, C-H. Lee ${ }^{\text {ah }}$, J. H. Lee ${ }^{c}$, W. Leight ${ }^{\text {v }}$, M. J. LeVine ${ }^{c}$, C. Li $^{\text {al }}$, N. Li ${ }^{\text {az }}, \mathrm{Y} \mathrm{Li}^{\text {ar }}, \mathrm{G}$. Lin ${ }^{\text {ba }}, \mathrm{S}$. J. Lindenbaum ${ }^{\mathrm{z}}$, M. A. Lisa ${ }^{\mathrm{ab}}$, F. Liu ${ }^{\text {az }}$, H. Liu ${ }^{\mathrm{e}}$, J. Liu ${ }^{\text {aj }}$, L. Liu ${ }^{\text {az }}$, T. Ljubicic ${ }^{\mathrm{c}}$,

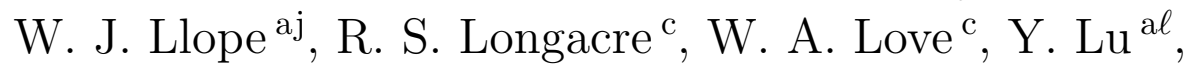
T. Ludlam ${ }^{\text {c }}$, G. L. Ma ${ }^{\text {an }}$, Y. G. Ma ${ }^{\text {an }}$, D. P. Mahapatra ${ }^{\ell}$, R. Majka ${ }^{\text {ba }}$, O. I. Mall ${ }^{\text {e }}$ L. K. Mangotra ${ }^{\mathrm{p}}$, R. Manweiler ${ }^{\text {at }}$, S. Margetis ${ }^{\mathrm{r}}$, C. Markert aq, H. Masui ${ }^{\text {u }}$, H. S. Matis ${ }^{\text {u, }}$ Yu. A. Matulenko af, D. McDonald ${ }^{\text {aj }}$, T. S. McShane ${ }^{\mathrm{i}}$, A. Meschanin af ${ }^{\text {af }}$ R. Millner ${ }^{\mathrm{v}}$, N. G. Minaev ${ }^{\text {af }}$, S. Mioduszewski ap ${ }^{\text {ap }}$ A. Mischke aa, B. Mohanty au, M.M. Mondal au, D. A. Morozov af, M. G. Munhoz ${ }^{\text {ak }}$, B. K. Nandi ${ }^{\mathrm{m}}$, C. Nattrass ${ }^{\text {ba }}$, T. K. Nayak ${ }^{\text {au }}$, J. M. Nelson ${ }^{\mathrm{b}}$, P. K. Netrakanti ${ }^{\text {ag }}$, M. J. $\mathrm{Ng}^{\mathrm{d}}$, L. V. Nogach ${ }^{\text {af }}$, S. B. Nurushev ${ }^{\text {af }}$, G. Odyniec ${ }^{\text {u }}$, A. Ogawa ${ }^{c}$, H. Okada $^{c}$, V. Okorokov ${ }^{\mathrm{y}}$, D. Olson ${ }^{\mathrm{u}}$, M. Pachr ${ }^{\mathrm{j}}$, B. S. Page ${ }^{\mathrm{n}}$, S. K. Pal ${ }^{\text {au }}$, Y. Pandit ${ }^{\text {r }}$, Y. Panebratsev ${ }^{\text {q }}$, T. Pawlak ${ }^{\text {av }}$, T. Peitzmann ${ }^{\text {aa }}$, V. Perevoztchikov ${ }^{\text {c }}$, C. Perkins ${ }^{\mathrm{d}}$, W. Peryt ${ }^{\text {av }}$, S. C. Phatak ${ }^{\ell}$, P. Pile ${ }^{\mathrm{c}}$, M. Planinic ${ }^{\mathrm{bb}}$, M. A. Ploskon ${ }^{\mathrm{u}}$, J. Pluta ${ }^{\text {av }}$, 
D. Plyku ac, N. Poljak ${ }^{\text {bb }}$, A. M. Poskanzer ${ }^{\text {u }}$,

B. V. K. S. Potukuchi ${ }^{\mathrm{p}}$, D. Prindle ${ }^{\text {aw }}$, C. Pruneau ${ }^{\text {ay }}$, N. K. Pruthi ${ }^{\text {ad }}$, P. R. Pujahari ${ }^{\mathrm{m}}$, J. Putschke ${ }^{\text {ba }}$, R. Raniwala ${ }^{\text {ai }}$, S. Raniwala ${ }^{\text {ai }}$, R. L. Ray ${ }^{\mathrm{aq}}, \mathrm{R}$. Redwine $^{\mathrm{v}}$, R. Reed ${ }^{\mathrm{e}}$,

A. Ridiger ${ }^{\mathrm{y}}, \mathrm{H}$. G. Ritter ${ }^{\mathrm{u}}$, J. B. Roberts ${ }^{\mathrm{aj}}$,

O. V. Rogachevskiy ${ }^{\mathrm{q}}$, J. L. Romero ${ }^{\mathrm{e}}$, A. Rose ${ }^{\mathrm{u}}$, C. Roy ${ }^{\mathrm{ao}}$,

L. Ruan ${ }^{\text {c}}$, M. J. Russcher aa, R. Sahoo ${ }^{\text {ao }}$, S. Sakai ${ }^{\mathrm{f}}$,

I. Sakrejda ${ }^{\text {u, T. Sakuma }}{ }^{\mathrm{v}}$, S. Salur u, J. Sandweiss ${ }^{\text {ba }}$,

M. Sarsour ap, J. Schambach aq, R. P. Scharenberg ag, N. Schmitz ${ }^{\mathrm{w}}$, J. Seger ${ }^{\mathrm{i}}$, I. Selyuzhenkov ${ }^{\mathrm{n}}$, P. Seyboth ${ }^{\mathrm{w}}$, A. Shabetai ${ }^{\circ}$, E. Shahaliev ${ }^{\text {q }}$, M. Shao ${ }^{\text {al }}$, M. Sharma ${ }^{\text {ay }}$, S. S. Shi ${ }^{\text {az }}$, X-H. Shi ${ }^{\text {an }}$, E. P. Sichtermann ${ }^{u}$, F. Simon ${ }^{w}$, R. N. Singaraju au, M.J. Skoby ag, N. Smirnov ${ }^{\text {ba }}$, P. Sorensen ${ }^{\text {c }}$,

J. Sowinski ${ }^{\mathrm{n}}$, H. M. Spinka ${ }^{\mathrm{a}}$, B. Srivastava ${ }^{\mathrm{ag}}$,

T. D. S. Stanislaus ${ }^{\text {at }}$, D. Staszak ${ }^{\mathrm{f}}$, M. Strikhanov ${ }^{\mathrm{y}}$, B. Stringfellow ${ }^{a g}$, A. A. P. Suaide ${ }^{a k}$, M. C. Suarez ${ }^{\text {, }}$, N. L. Subba ${ }^{r}$, M. Sumbera ${ }^{k}, X . M . \operatorname{Sun}^{u}$, Y. $\operatorname{Sun}^{2 \ell}$, Z. Sun ${ }^{\mathrm{t}}$, B. Surrow ${ }^{v}$, T. J. M. Symons ${ }^{u}$, A. Szanto de Toledo ak, J. Takahashi ${ }^{\mathrm{g}}$, A. H. Tang ${ }^{\mathrm{c}}$, Z. Tang ${ }^{\mathrm{a}}$, L. H. Tarini ${ }^{\text {ay }}$, T. Tarnowsky ${ }^{\mathrm{x}}$, D. Thein ${ }^{\mathrm{aq}}$, J. H. Thomas ${ }^{\mathrm{u}}$, J. Tian ${ }^{\text {an }}$, A. R. Timmins ${ }^{\text {ay }}$, S. Timoshenko ${ }^{\mathrm{y}}$, D. Tlusty ${ }^{\mathrm{k}}$, M. Tokarev ${ }^{\mathrm{q}}$, T. A. Trainor ${ }^{\text {aw }}$, V.N. Tram ${ }^{\text {u}}$, A. L. Trattner ${ }^{d}$, S. Trentalange ${ }^{f}$,

R. E. Tribble ${ }^{\text {ap }}$, O. D. Tsai ${ }^{\mathrm{f}}$, J. Ulery ${ }^{\mathrm{ag}}$, T. Ullrich ${ }^{\mathrm{c}}$, D. G. Underwood ${ }^{\text {a }}$, G. Van Buren ${ }^{\text {c }}$, M. van Leeuwen ${ }^{\text {aa }}$, G. van Nieuwenhuizen ${ }^{\mathrm{v}}$, J.A. Vanfossen,Jr. ${ }^{\mathrm{r}}$, R. Varma $^{\mathrm{m}}$, G. M. S. Vasconcelos ${ }^{\mathrm{g}}$, A. N. Vasiliev ${ }^{\text {af }}$, F. Videbaek ${ }^{\mathrm{c}}$, S. E. Vigdor ${ }^{n}$, Y.P. Viyogi ${ }^{\ell}$, S. Vokal ${ }^{q}$, S. A. Voloshin ${ }^{\text {ay }}$, M. Wada ${ }^{\text {aq }}$, M. Walker ${ }^{\mathrm{v}}$, F. Wang ${ }^{\mathrm{ag}}$, G. Wang ${ }^{\mathrm{f}}$, H. Wang ${ }^{\mathrm{x}}$, J. S. Wang ${ }^{\mathrm{t}}$, Q. Wang ${ }^{\mathrm{ag}}$, X. Wang ${ }^{\mathrm{ar}}$, X. L. Wang ${ }^{\mathrm{a} \ell}$, Y. Wang ${ }^{\text {ar }}$,

G. Webb ${ }^{\text {s, J. C. Webb }}{ }^{\text {at }}$, G. D. Westfall ${ }^{\mathrm{x}}$, C. Whitten Jr. ${ }^{\mathrm{f}}$, H. Wieman ${ }^{\text {u }}$, S. W. Wissink ${ }^{\mathrm{n}}$, R. Witt ${ }^{\text {as }}, \mathrm{Y} . \mathrm{Wu}^{\mathrm{az}}, \mathrm{W}$. Xie ${ }^{\mathrm{ag}}$, N. Xu ${ }^{u}$, Q. H. Xu ${ }^{\text {am }}, Y . X^{a}{ }^{a \ell}, Z$ Z. Xu ${ }^{c}, Y$. Yang ${ }^{t}$, P. Yepes ${ }^{a j}$, K. Yip ${ }^{c}$, I-K. Yoo ${ }^{\text {ah }}$, Q. Yue $^{\text {ar }}$, M. Zawisza ${ }^{\text {av }}$, H. Zbroszczyk ${ }^{\text {av }}$,

W. Zhan ${ }^{\mathrm{t}}$, S. Zhang an, W. M. Zhang ${ }^{\mathrm{r}}$, X.P. Zhang ${ }^{\mathrm{u}}$, Y. Zhang ${ }^{\text {u }}$ Z. P. Zhang ${ }^{a \ell}$, Y. Zhao $^{\text {al }}$, C. Zhong an, J. Zhou ${ }^{\text {aj }}$, 


\section{Zhu ${ }^{\text {ar }}$, R. Zoulkarneev ${ }^{\mathrm{q}}$, Y. Zoulkarneeva ${ }^{\mathrm{q}}$, and J. X. Zuo ${ }^{\text {an }}$, (STAR Collaboration)}

a Argonne National Laboratory, Argonne, Illinois 60439

${ }^{\mathrm{b}}$ University of Birmingham, Birmingham, United Kingdom

${ }^{\mathrm{c}}$ Brookhaven National Laboratory, Upton, New York 11973

${ }^{\mathrm{d}}$ University of California, Berkeley, California 94720

e University of California, Davis, California 95616

${ }^{\mathrm{f}}$ University of California, Los Angeles, California 90095

' Universidade Estadual de Campinas, Sao Paulo, Brazil

${ }^{\mathrm{h}}$ University of Illinois at Chicago, Chicago, Illinois 6060'7

${ }^{\mathrm{i}}$ Creighton University, Omaha, Nebraska 68178

${ }^{\mathrm{j}}$ Czech Technical University in Prague, FNSPE, Prague, 115 19, Czech Republic

${ }^{\mathrm{k}}$ Nuclear Physics Institute AS CR, 25068 Řez/Prague, Czech Republic

${ }^{\ell}$ Institute of Physics, Bhubaneswar 751005, India

${ }^{\mathrm{m}}$ Indian Institute of Technology, Mumbai, India

${ }^{\mathrm{n}}$ Indiana University, Bloomington, Indiana 47408

${ }^{\circ}$ Institut de Recherches Subatomiques, Strasbourg, France

p University of Jammu, Jammu 180001, India

${ }^{\mathrm{q}}$ Joint Institute for Nuclear Research, Dubna, 141 980, Russia

${ }^{\mathrm{r}}$ Kent State University, Kent, Ohio 44242

${ }^{\mathrm{s}}$ University of Kentucky, Lexington, Kentucky, 40506-0055

${ }^{\mathrm{t}}$ Institute of Modern Physics, Lanzhou, China

u Lawrence Berkeley National Laboratory, Berkeley, California 94720

${ }^{\mathrm{v}}$ Massachusetts Institute of Technology, Cambridge, MA 02139-4307

${ }^{\mathrm{w}}$ Max-Planck-Institut für Physik, Munich, Germany

${ }^{x}$ Michigan State University, East Lansing, Michigan 48824

${ }^{\mathrm{y}}$ Moscow Engineering Physics Institute, Moscow Russia

${ }^{z}$ City College of New York, New York City, New York 10031

aa NIKHEF and Utrecht University, Amsterdam, The Netherlands

${ }^{\text {ab }}$ Ohio State University, Columbus, Ohio 43210

${ }^{a c}$ Old Dominion University, Norfolk, VA, 23529

ad Panjab University, Chandigarh 160014, India

${ }^{a e}$ Pennsylvania State University, University Park, Pennsylvania 16802

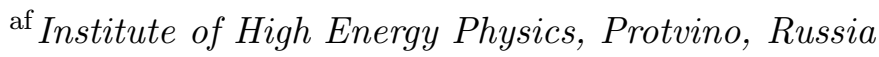

ag Purdue University, West Lafayette, Indiana 47907

${ }^{\text {ah }}$ Pusan National University, Pusan, Republic of Korea 
ai University of Rajasthan, Jaipur 302004, India

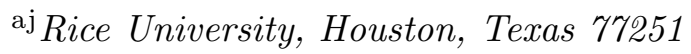

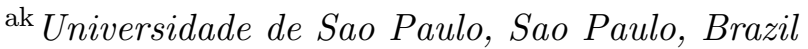

${ }^{a}$ University of Science 83 Technology of China, Hefei 230026, China

${ }^{a m}$ Shandong University, Jinan, Shandong 250100, China

${ }^{\text {an }}$ Shanghai Institute of Applied Physics, Shanghai 201800, China

ao $S U B A T E C H$, Nantes, France

ap Texas A\&M University, College Station, Texas 77843

${ }^{a q}$ University of Texas, Austin, Texas 78712

ar Tsinghua University, Beijing 100084, China

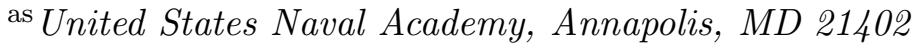

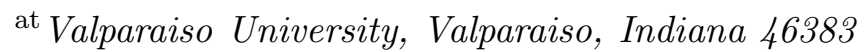

${ }^{\mathrm{au}}$ Variable Energy Cyclotron Centre, Kolkata 700064, India

${ }^{a v}$ Warsaw University of Technology, Warsaw, Poland

${ }^{\text {aw }}$ University of Washington, Seattle, Washington 98195

ay Wayne State University, Detroit, Michigan 48201

az Institute of Particle Physics, CCNU (HZNU), Wuhan 430079, China

${ }^{\text {ba }}$ Yale University, New Haven, Connecticut 06520

${ }^{\mathrm{bb}}$ University of Zagreb, Zagreb, HR-10002, Croatia

\begin{abstract}
We present the multiplicity and pseudorapidity distributions of photons produced in $\mathrm{Au}+\mathrm{Au}$ and $\mathrm{Cu}+\mathrm{Cu}$ collisions at $\sqrt{s_{\mathrm{NN}}}=62.4$ and $200 \mathrm{GeV}$. The photons are measured in the region $-3.7<\eta<-2.3$ using the photon multiplicity detector in the STAR experiment at RHIC. The number of photons produced per average number of participating nucleon pairs increases with the beam energy and is independent of the collision centrality. For collisions with similar average numbers of participating nucleons the photon multiplicities are observed to be similar for $\mathrm{Au}+\mathrm{Au}$ and $\mathrm{Cu}+\mathrm{Cu}$ collisions at a given beam energy. The ratios of the number of charged particles to photons in the measured pseudorapidity range are found to be $1.4 \pm 0.1$ and $1.2 \pm 0.1$ for $\sqrt{s_{\mathrm{NN}}}=62.4 \mathrm{GeV}$ and $200 \mathrm{GeV}$, respectively. The energy dependence of this ratio could reflect varying contributions from baryons to charged particles, while mesons are the dominant contributors to photon production in the given kinematic region. The photon pseudorapidity distributions normalized by average number of participating nucleon pairs, when plotted as a function of $\eta-y_{\text {beam }}$, are found to follow a longitudinal scaling independent of centrality and colliding ion species at both beam energies.
\end{abstract}

Key words: Particle production, photons, forward rapidity, limiting fragmentation 


\section{Introduction}

For high energy heavy-ion collisions, measurements of particle multiplicity provide information on particle production mechanisms [1]. Event-by-event fluctuations in the multiplicity of produced particles within a thermodynamic picture could be related to matter compressibility [2]. The event-by-event correlation between photon and charged particle multiplicities can be used to test the predictions of formation of disoriented chiral condensates [3]. The variation of particle density in pseudorapidity $(\eta)$ with collision centrality can shed light on the relative contribution of soft and hard (perturbative QCD jets) processes in particle production [4]. Multiplicity measurements can provide tests of ideas on initial conditions in heavy-ion collisions based on parton saturation [5] and color glass condensates [6]. Under certain model assumptions, the particle density in pseudorapidity can provide information on the initial temperature and velocity of sound in the medium [7]. The pseudorapidity distributions are found to be sensitive to the effects of re-scattering, hadronic final-state interactions, and longitudinal flow [8].

Several interesting features of the dependence of particle density in pseudorapidity have been observed in $\mathrm{Au}+\mathrm{Au}$ collisions from the experiments at the Relativistic Heavy-Ion Collider (RHIC). Particle production is found to follow a unique, collision energy independent, longitudinal scaling [9] in $p+p$ and $d+\mathrm{Au}$, as well as in heavy-ion collisions [10,11]. Such longitudinal scaling is also found to be independent of collision centrality for photons $[12,13]$. The total charged particle multiplicity (integrated over the full pseudorapidity range) per average number of participating nucleon $\left(\left\langle N_{\text {part }}\right\rangle\right)$ pair is found to be independent of collision centrality [10]. However, at mid-rapidity $(|\eta|<1)$, charged particle multiplicity per $\left\langle N_{\text {part }}\right\rangle$ is observed to increase from peripheral to central collisions [10]. This clearly indicates that the mechanism of particle production could be different in different pseudorapidity regions. In the year 2005, a unique opportunity to investigate the system-size dependence of global observables occurred when $\mathrm{Cu}+\mathrm{Cu}$ collisions were produced at $\mathrm{RHIC}$. In light of the earlier results of photon multiplicity scaling with $\left\langle N_{\text {part }}\right\rangle[12,13]$ at forward rapidity, one could make direct comparison of the observables $\left(N_{\gamma}\right.$ and $\left.d N_{\gamma} / d \eta\right)$ for $\mathrm{Cu}+\mathrm{Cu}$ and $\mathrm{Au}+\mathrm{Au}$ collisions for systems having similar values of $\left\langle N_{\text {part }}\right\rangle$.

In this paper we present the first measurements of photon multiplicity distributions at forward rapidities in $\mathrm{Cu}+\mathrm{Cu}$ collisions at $\sqrt{s_{\mathrm{NN}}}=62.4$ and $200 \mathrm{GeV}$ and $\mathrm{Au}+\mathrm{Au}$ collisions at $\sqrt{s_{\mathrm{NN}}}=200 \mathrm{GeV}$ from the STAR experiment [14] at RHIC. The results from $\mathrm{Au}+\mathrm{Au}$ collisions at $\sqrt{s_{\mathrm{NN}}}=62.4 \mathrm{GeV}$ were re- 
ported in Refs. [12,13]. The photon multiplicity measurements are presented for various collision centrality classes and are compared to corresponding results for charged particles. The photon production is dominated by those from the decay of $\pi^{0} \mathrm{~S}$ [12]. HIJING [15] calculations indicate that about $93-96 \%$ of photons are from inclusive $\pi^{0}$ decays for the $\sqrt{s_{\mathrm{NN}}}$ and $\eta$ range studied.

\section{Experiment and Analysis}

The STAR detector contains several subsystems which measure hadronic and electromagnetic observables at forward rapidity [14]. The main subsystem used in the present analysis is the Photon Multiplicity Detector (PMD) [17]. Photons are detected using a highly granular preshower PMD located $-5.4 \mathrm{~m}$ from the center of the Time Projection Chamber (TPC), the nominal collision point, along the beam axis. The measurements are carried out within the pseudorapidity region of -3.7 to -2.3 at $\sqrt{s_{\mathrm{NN}}}=62.4$ and $200 \mathrm{GeV}$ for $\mathrm{Au}+\mathrm{Au}$ and $\mathrm{Cu}+\mathrm{Cu}$ collisions. A minimum bias trigger is obtained using the charged particle hits from the Central Trigger Barrel (CTB), an array of scintillator slats arranged in a barrel around the TPC, two Zero Degree Hadronic Calorimeters (ZDCs) located $\pm 18 \mathrm{~m}$ from the center of the TPC, and two Beam-Beam Counters (BBCs) [18]. A total of 307k, 334k, 289k and 330k minimum bias events for $\mathrm{Au}+\mathrm{Au} 200, \mathrm{Au}+\mathrm{Au} 62.4, \mathrm{Cu}+\mathrm{Cu} 200$ and $\mathrm{Cu}+\mathrm{Cu} 62.4$ $\mathrm{GeV}$ collisions, respectively, were analyzed. These events have a collision vertex position less than $30 \mathrm{~cm}$ from the center of the TPC along the beam axis. The centrality determination in this analysis uses the uncorrected multiplicity of charged particles in the region $|\eta|<0.5$, as measured in the TPC. The average number of participating nucleons is obtained from Monte Carlo Glauber

calculations [19]. The $\left\langle N_{\text {part }}\right\rangle$ values corresponding to various percentages of the cross section for $\mathrm{Au}+\mathrm{Au}$ and $\mathrm{Cu}+\mathrm{Cu}$ collisions at $\sqrt{s_{\mathrm{NN}}}=62.4$ and 200 $\mathrm{GeV}$ are given in the Table 1 .

The PMD consists of two planes (charged particle veto and preshower) of arrays of cellular gas proportional counters. A lead plate (3 radiation lengths thickness) is placed between the two planes and serves as a photon converter. The sensitive medium is a gas mixture of $\mathrm{Ar}$ and $\mathrm{CO}_{2}$ in the ratio 70:30 by weight. There are 41,472 hexagonal cells in each plane, which are located inside 12 high voltage insulated and gas-tight chambers called supermodules. To each supermodule is applied a negative voltage of $1400 \mathrm{~V}$ as the operating voltage. A photon traversing the converter produces an electromagnetic shower in the preshower plane, leading to a large signal, spread over several cells. In contrast, a charged particle's signal is essentially confined to a single cell. The photon conversion efficiency studied from simulations is found to increase with increasing photon energy $\left(E_{\gamma}\right)$ up to $1 \mathrm{GeV}$ and then saturate for higher energies. The typical values for the three radiation length converter 
Table 1

Average number of participating nucleons $\left(\left\langle N_{\text {part }}\right\rangle\right)$ for various collision centralities for $\mathrm{Au}+\mathrm{Au}$ and $\mathrm{Cu}+\mathrm{Cu}$ collisions at $\sqrt{s_{\mathrm{NN}}}=62.4$ and $200 \mathrm{GeV}$.

\begin{tabular}{|c|c|c|c|c|}
\hline \% cross section & $\begin{array}{c}\left\langle N_{\text {part }}^{\mathrm{AuAu}}\right\rangle \\
200 \mathrm{GeV}\end{array}$ & $\begin{array}{c}\left\langle N_{\text {part }}^{\mathrm{AuAu}}\right\rangle \\
62.4 \mathrm{GeV}\end{array}$ & $\begin{array}{c}\left\langle N_{\text {part }}^{\mathrm{CuCu}}\right\rangle \\
200 \mathrm{GeV}\end{array}$ & $\begin{array}{c}\left\langle N_{\text {part }}^{\mathrm{CuCu}}\right\rangle \\
62.4 \mathrm{GeV}\end{array}$ \\
\hline $0-5$ & $352.4_{-4.0}^{+3.4}$ & $347.3_{-3.7}^{+4.3}$ & - & - \\
$0-10$ & $325.9_{-4.3}^{+5.5}$ & - & $99.0_{-1.2}^{+1.5}$ & $96.4_{-2.6}^{+1.1}$ \\
$5-10$ & $299.3_{-6.7}^{+6.6}$ & $293.3_{-5.6}^{+7.3}$ & - & - \\
$10-20$ & $234.5_{-7.8}^{+9.1}$ & $229.0_{-7.7}^{+9.2}$ & $74.6_{-1.0}^{+1.3}$ & $72.2_{-1.9}^{+0.6}$ \\
$20-30$ & $166.6_{-9.6}^{+10.1}$ & $162.0_{-9.5}^{+10.0}$ & $53.7_{-0.7}^{+1.0}$ & $51.8_{-1.2}^{+0.5}$ \\
$30-40$ & $115.5_{-9.6}^{+9.6}$ & $112.0_{-9.1}^{+9.6}$ & $37.8_{-0.5}^{+0.7}$ & $36.2_{-0.8}^{+0.4}$ \\
$40-50$ & $76.7_{-9.1}^{+9.0}$ & $74.2_{-8.5}^{+9.0}$ & $26.2_{-0.4}^{+0.5}$ & $24.9_{-0.6}^{+0.4}$ \\
$50-60$ & $47.3_{-8.1}^{+7.6}$ & $45.8_{-7.1}^{+7.0}$ & $17.2_{-0.2}^{+0.4}$ & $16.3_{-0.3}^{+0.4}$ \\
$60-70$ & $26.9_{-6.5}^{+5.5}$ & $25.9_{-5.6}^{+5.6}$ & - & - \\
$70-80$ & $14.1_{-4.0}^{+3.6}$ & $13.0_{-4.6}^{+3.4}$ & - & - \\
\hline
\end{tabular}

are observed to be around $70 \%$ for $E_{\gamma}=0.2 \mathrm{GeV}$ and $95 \%$ for $E_{\gamma}=1 \mathrm{GeV}$. In the present analysis, only the data from the preshower plane are used. Further details of the design and characteristics of the PMD are found in Ref. [17].

The analysis of the data from the PMD involves the following: (a) event selection, (b) cell-to-cell gain calibration, and (c) reconstruction or extraction of the photon multiplicity. The cell-to-cell gain calibration is done using the ADC distributions of isolated cells (cells with six neighbouring cells having zero ADC). The ADC distribution of an isolated cell is treated as the response of the cell to charged particles, corresponding to a minimum ionizing particle (MIP) [17]. For most of the cells this response follows a Landau distribution. We use the mean of the ADC distribution of isolated cells to estimate and correct the relative gains of all cells within each supermodule. The cell-to-cell gain variation is between $10-25 \%$ for different supermodules. The extraction of the photon multiplicity proceeds in two steps involving clustering of hits and photon-hadron discrimination. Hit clusters consist of contiguous cell signals. Photons are separated from charged particles using the following conditions: (a) the number of cells in a cluster is $>1$, and (b) the cluster signal is larger than 3 times the average MIP response of all isolated cells in a supermodule. The choice of the conditions is based on results of detailed simulations $[12,13,17]$. The number of selected clusters, called $\gamma$-like clusters $\left(N_{\gamma \text {-like }}\right)$, in different supermodules for the same $\eta$ coverage are used to evaluate the effect of possible non-uniformity in the response of the detector. 

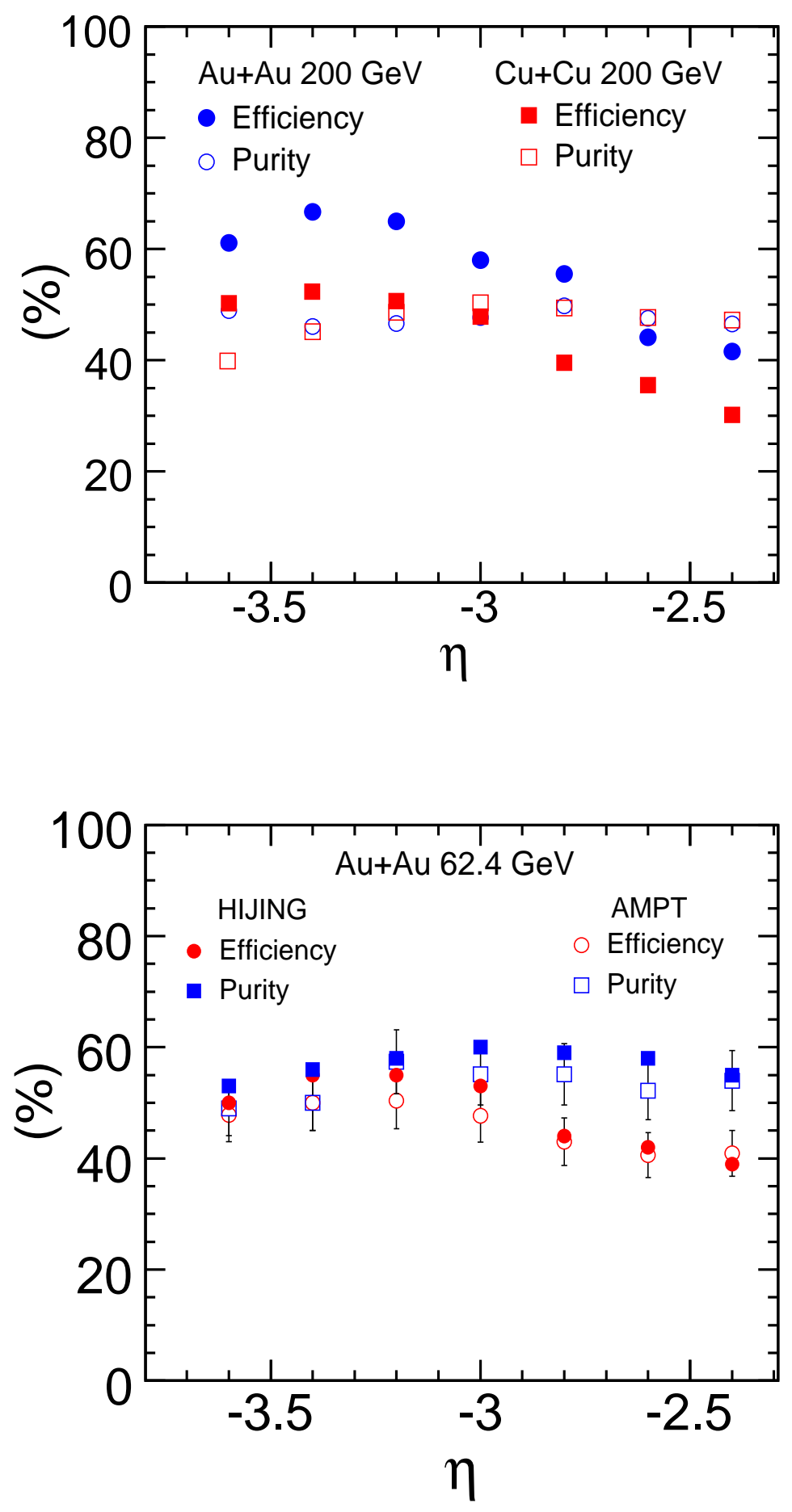

Fig. 1. (color online) Top panel: Photon reconstruction efficiency $\left(\epsilon_{\gamma}\right)$ (solid symbols) and purity of photon sample $\left(f_{\mathrm{p}}\right)$ (open symbols) for PMD as a function of pseudorapidity $(\eta)$ for minimum bias $\mathrm{Au}+\mathrm{Au}$ and $\mathrm{Cu}+\mathrm{Cu}$ at $\sqrt{s_{\mathrm{NN}}}=200 \mathrm{GeV}$. Bottom panel: Comparison between estimated $\epsilon_{\gamma}$ and $f_{\mathrm{p}}$ for PMD as a function of $\eta$ for minimum bias $\mathrm{Au}+\mathrm{Au}$ at $\sqrt{s_{\mathrm{NN}}}=62.4 \mathrm{GeV}$ using HIJING and AMPT models. The error bars on the AMPT data are statistical and those for HIJING are within the symbol size. 
To estimate the number of photons $\left(N_{\gamma}\right)$ from the detected $N_{\gamma-\text { like }}$ clusters, we use the photon reconstruction efficiency $\left(\epsilon_{\gamma}\right)$ and purity $\left(f_{\mathrm{p}}\right)$ of the $\gamma$-like sample defined $[12,20]$ as $\epsilon_{\gamma}=N_{\mathrm{cls}}^{\gamma, \text { th }} / N_{\gamma}$ and $f_{\mathrm{p}}=N_{\mathrm{cls}}^{\gamma, \text { th }} / N_{\gamma-\text { like }}$, respectively. $N_{c l s}^{\gamma, \text { th }}$ is the number of photon clusters above the photon-hadron discriminator threshold. The photon multiplicity in data is then obtained as $N_{\gamma}=\left(f_{\mathrm{p}} / \epsilon_{\gamma}\right) N_{\gamma-\text { like }}[12,17,20]$, where the ratio $f_{\mathrm{p}} / \epsilon_{\gamma}$ is estimated from simulations as described below. Both $\epsilon_{\gamma}$ and $f_{\mathrm{p}}$ are obtained from a detailed Monte Carlo simulation using HIJING version 1.382 [15] with default parameter settings and the detector simulation package GEANT [21], which incorporates the full STAR detector framework. In our previous work [13] it has been shown that HIJING reproduces the $N_{\mathrm{ch}} / N_{\gamma}$ ratio in $\mathrm{Au}+\mathrm{Au}$ collisions at $\sqrt{s_{\mathrm{NN}}}=62.4$ $\mathrm{GeV}$. For estimation of $\epsilon_{\gamma}$ in simulations it may be important to know the inclusive photon $p_{\mathrm{T}}$ distribution. Due to lack of experimental measurement of the inclusive photon $p_{T}$ distribution at forward rapidity it assumed that they are similar to those from HIJING model. In order to investigate the possible differences, $\epsilon_{\gamma}$ and $f_{\mathrm{p}}$ are also obtained from a detailed Monte Carlo simulation using AMPT model [16] with default parameter settings. The AMPT model is a multiphase transport model which includes both initial partonic and final hadronic interactions. The differences between the $\epsilon_{\gamma}$ and $f_{\mathrm{p}}$ values estimated using the two models are less than $5 \%$. This difference is attributed to systematic errors on $N_{\gamma}$. Both $\epsilon_{\gamma}$ and $f_{\mathrm{p}}$ can vary with pseudorapidity and centrality. The $\epsilon_{\gamma}$ and $f_{\mathrm{p}}$ for minimum bias $\mathrm{Au}+\mathrm{Au}$ and $\mathrm{Cu}+\mathrm{Cu}$ at $200 \mathrm{GeV}$ are shown in top panel of Fig. 1. The photon reconstruction efficiency (which includes the detector acceptance corrections) varies from $30 \%$ at $\eta=-2.3$ to $60 \%$ at $\eta=-3.7$ for all collision centralities obtained from simulations for $\mathrm{Au}+\mathrm{Au}$ and $\mathrm{Cu}+\mathrm{Cu}$ collisions at $62.4[12,13]$ and $200 \mathrm{GeV}$. The purity of the photon sample is nearly constant as a function of $\eta$ and varies between $40 \%$ and $60 \%$ for $\mathrm{Au}+\mathrm{Au}$ and $\mathrm{Cu}+\mathrm{Cu}$ collisions at $62.4[12,13]$ and $200 \mathrm{GeV}$. Both $\epsilon_{\gamma}$ and $f_{\mathrm{p}}$ show slight variation with system-size. The $\eta$ dependence of the $\epsilon_{\gamma}$ reflects mainly the varying detector acceptance between $\eta=-2.0$ and $\eta=-3.0$. There is also a small effect on the $\eta$ on $\epsilon_{\gamma}$ due to varying particle density as a function of $\eta$. This is effect is already reflected in the comparison of $\epsilon_{\gamma}$ values between $\mathrm{Au}+\mathrm{Au}$ and $\mathrm{Cu}+\mathrm{Cu}$. The $f_{\mathrm{p}}$ values by definition are not affected by detector acceptance. The bottom panel of Fig. 1 shows a typical comparison of estimated $\epsilon_{\gamma}$ and $f_{\mathrm{p}}$ using HIJING and AMPT models for $\mathrm{Au}+\mathrm{Au}$ minimum bias collisions at $62.4 \mathrm{GeV}$. The differences are within $5 \%$ level. The systematic errors are discussed below. The lower limit of photon $p_{\mathrm{T}}$ acceptance in the PMD is estimated from detector simulations to be 20 $\mathrm{MeV} / c$.

The systematic errors for photon multiplicity $\left(N_{\gamma}\right)$ are due to $[12,13]$ (a) uncertainty in estimates of $\epsilon_{\gamma}$ and $f_{\mathrm{p}}$ values arising from splitting of clusters, the choice of photon-hadron discriminator threshold and choice of different event generators for their estimation and (b) uncertainty in $N_{\gamma}$ arising from the non-uniformity of the detector response (primarily due to cell-to-cell gain 

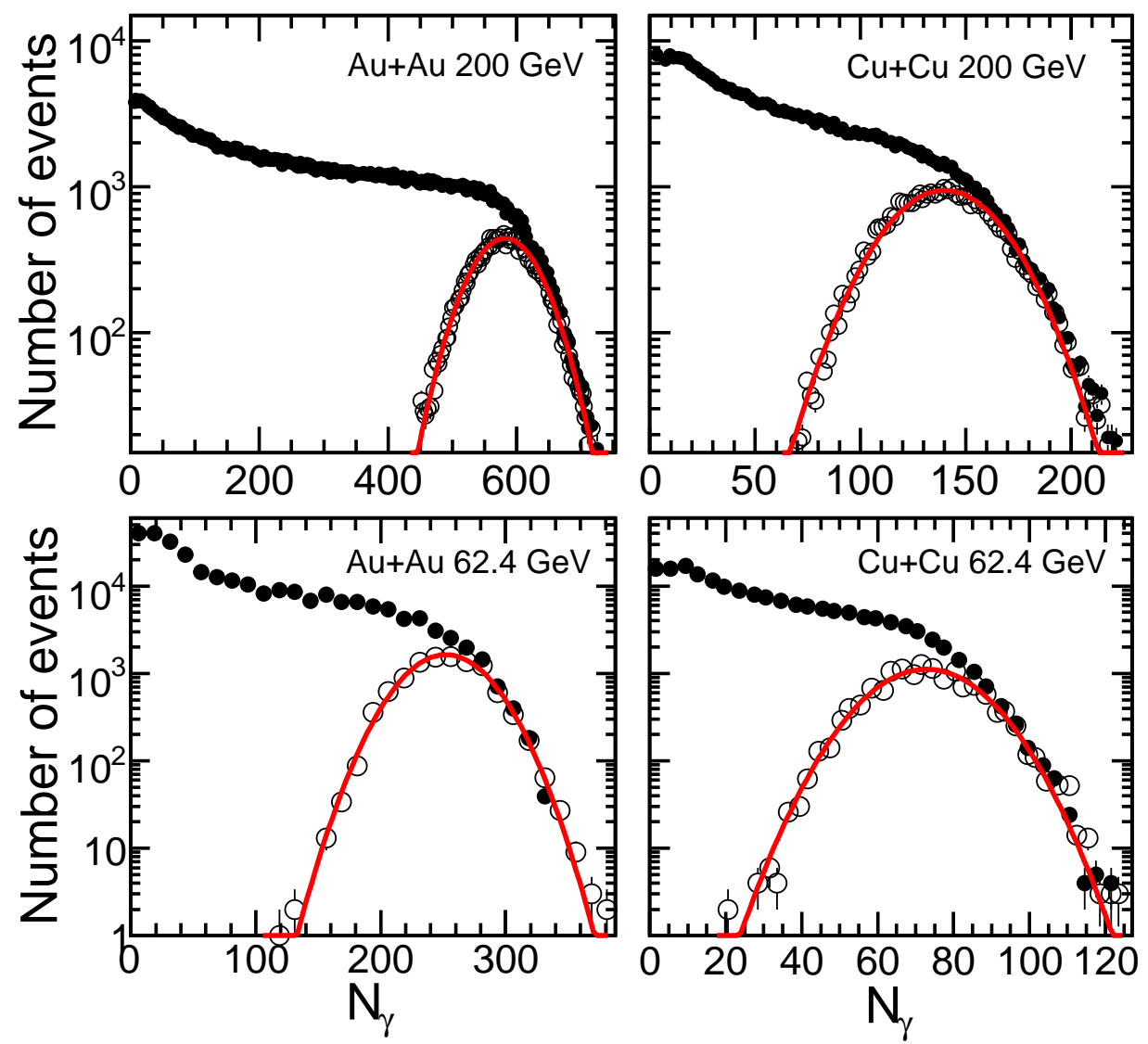

Fig. 2. (color online) Event-by-event photon multiplicity distributions (solid circles) for $\mathrm{Au}+\mathrm{Au}$ and $\mathrm{Cu}+\mathrm{Cu}$ at $\sqrt{s_{\mathrm{NN}}}=62.4$ and $200 \mathrm{GeV}$. The distributions for top 0-5\% central $\mathrm{Au}+\mathrm{Au}$ collisions and top $0-10 \%$ central $\mathrm{Cu}+\mathrm{Cu}$ collisions are also shown (open circles). The photon multiplicity distributions for central collisions are observed to be Gaussian (solid line). Only statistical errors are shown.

variation). The error in $N_{\gamma}$ due to (a) is estimated from Monte Carlo simulations to be $\leq 16 \%$ for all systems and beam energies studied. It is fairly independent of collision centrality. The error on $N_{\gamma}$ due to (b) is estimated using average gains for normalization and by studying the azimuthal dependence of the photon density, in an $\eta$ window in the detector, and is found to be $\sim 10 \%$. The total systematic error in $N_{\gamma}$ is $\sim 19 \%$ for both central and peripheral collisions and is similar for $\mathrm{Au}+\mathrm{Au}$ and $\mathrm{Cu}+\mathrm{Cu}$ at 62.4 and 200 $\mathrm{GeV}$. The statistical errors are small and within the symbol size for the results shown in the figures. 
Table 2

Gaussian fit parameters for photon multiplicity distributions for $-3.7<\eta<-2.3$ for central $\mathrm{Au}+\mathrm{Au}(0-5 \%)$ and $\mathrm{Cu}+\mathrm{Cu}(0-10 \%)$ at $\sqrt{s_{\mathrm{NN}}}=62.4$ and $200 \mathrm{GeV}$.

\begin{tabular}{|c|c|c|c|}
\hline Collision Type & $\left\langle N_{\text {part }}\right\rangle$ & $\left\langle N_{\gamma}\right\rangle$ & $\sigma_{\gamma}$ \\
\hline $\mathrm{Au}+\mathrm{Au} 62.4 \mathrm{GeV}$ & 347.3 & 252 & 30 \\
\hline $\mathrm{Au}+\mathrm{Au} 200 \mathrm{GeV}$ & 352.4 & 582 & 52 \\
\hline $\mathrm{Cu}+\mathrm{Cu} 62.4 \mathrm{GeV}$ & 96.4 & 73 & 13 \\
\hline $\mathrm{Cu}+\mathrm{Cu} 200 \mathrm{GeV}$ & 99.0 & 140 & 26 \\
\hline
\end{tabular}

\section{$3 \quad$ Multiplicity Distributions}

Figure 2 shows the photon multiplicity distributions for minimum bias $\mathrm{Au}+\mathrm{Au}$ and $\mathrm{Cu}+\mathrm{Cu}$ collisions at 62.4 and $200 \mathrm{GeV}$. The distributions for both energies and colliding ion species show a characteristic shape with a rise at small multiplicity owing to peripheral events. This indicates the probability of occurrence of peripheral collisions is higher. This rise is followed by a near plateau region with increasing photon multiplicity. This region is more prominent for $\mathrm{Au}+\mathrm{Au}$ than $\mathrm{Cu}+\mathrm{Cu}$. It corresponds to mid-central collisions. There is a falloff region in the distributions for the most central collisions. The shape of the distribution in the fall-off region is governed by intrinsic fluctuations in the measured quantity and on the limited acceptance. Also shown in Fig. 2 are event-by-event photon multiplicity distributions for central $\mathrm{Au}+\mathrm{Au}(0$ $5 \%)$ and $\mathrm{Cu}+\mathrm{Cu}(0-10 \%)$ at $\sqrt{s_{\mathrm{NN}}}=62.4$ and $200 \mathrm{GeV}$. The solid lines are Gaussian fits to the data. The fit parameters are given in Table 2.

Figure 3 shows the pseudorapidity distributions of photons measured in the $\mathrm{PMD}$ for various collision centralities in $\mathrm{Au}+\mathrm{Au}$ and $\mathrm{Cu}+\mathrm{Cu}$ at $\sqrt{s_{\mathrm{NN}}}=62.4$ and $200 \mathrm{GeV}$. As expected, the photon yield increases with decreasing $|\eta|$. The photon multiplicity is found to increase from peripheral to central collisions. Comparisons to HIJING calculations for central (0-5\% for $\mathrm{Au}+\mathrm{Au}$ and 0$10 \%$ for $\mathrm{Cu}+\mathrm{Cu}$ ) and $30-40 \%$ mid-central collisions are also shown in the figure (solid curves). The HIJING results are in reasonable agreement with the data for both beam energies and colliding ion species. Similar conclusions are drawn for other centrality classes as well. 

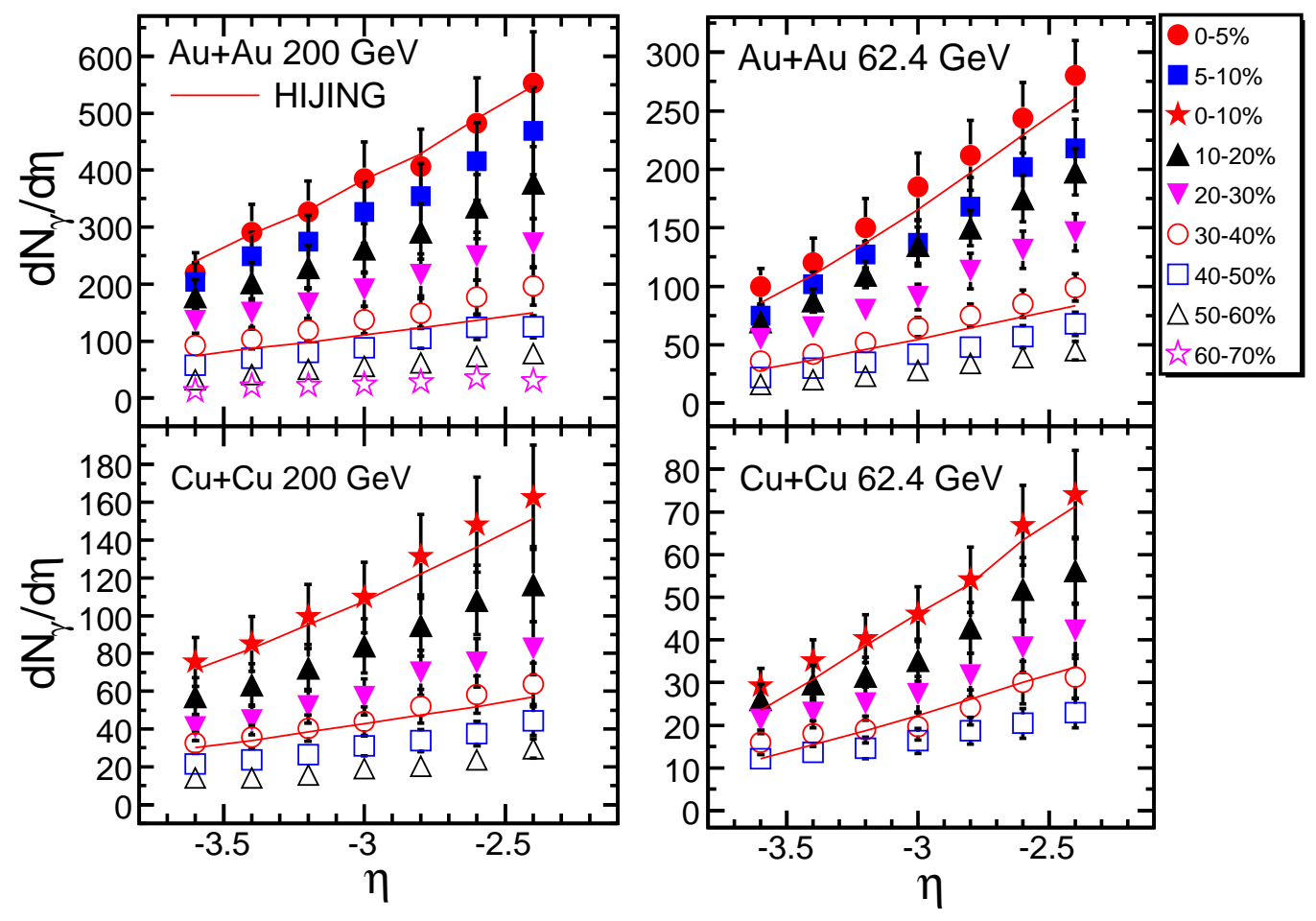

Fig. 3. (color online) Photon pseudorapidity distributions for $\mathrm{Au}+\mathrm{Au}$ and $\mathrm{Cu}+\mathrm{Cu}$ at $\sqrt{s_{\mathrm{NN}}}=62.4$ and $200 \mathrm{GeV}$. The results for several centrality classes are shown. The solid curves are results of HIJING simulations for central (0-5\% for $\mathrm{Au}+\mathrm{Au}$ and $0-10 \%$ for $\mathrm{Cu}+\mathrm{Cu}$ ) and $30-40 \%$ mid-central collisions. The errors shown are systematic, statistical errors are negligible in comparison.

\section{Scaling of Photon Production}

\subsection{Scaling with $\left\langle N_{\text {part }}\right\rangle$}

The scaling of particle multiplicity with $\left\langle N_{\text {part }}\right\rangle$ indicates the dominance of soft processes in particle production at RHIC, whereas scaling with average number of binary collisions $\left(\left\langle N_{\text {bin }}\right\rangle\right)$ indicates the onset of hard processes (pQCD jets). The PHENIX experiment first showed that at mid-rapidity, the charged particle production scales with a combination of $\left\langle N_{\text {part }}\right\rangle$ and $\left\langle N_{\text {bin }}\right\rangle$ [4], indicating significant contribution of hard processes in particle production. The PHOBOS experiment showed that such scaling has a pseudorapidity dependence [10]. At mid-rapidity $(|\eta|<1)$ particle production scales with a combination of $\left\langle N_{\text {part }}\right\rangle$ and $\left\langle N_{\text {bin }}\right\rangle$; for the range $3<|\eta|<3.4$ it scales with $\left\langle N_{\text {part }}\right\rangle$; and for the region $5<|\eta|<5.4$, the particle production per average number of participating nucleon pair decreases with increasing $\left\langle N_{\text {part }}\right\rangle$. 

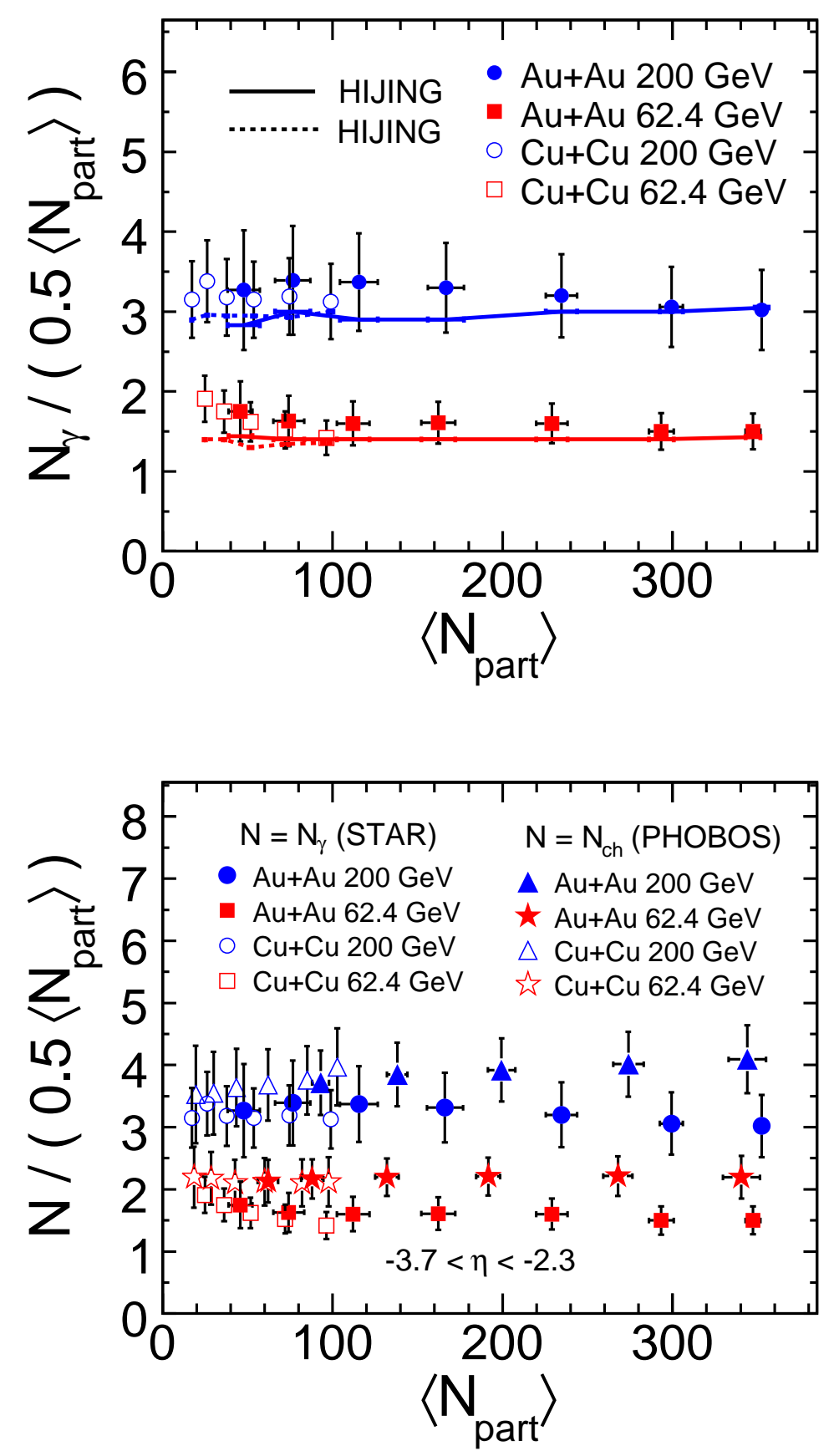

Fig. 4. (color online) Top panel: The number of photons divided by $\left\langle N_{\text {part }}\right\rangle / 2$ as a function of average number of participating nucleons for $\mathrm{Au}+\mathrm{Au}$ and $\mathrm{Cu}+\mathrm{Cu}$ at $\sqrt{s_{\mathrm{NN}}}=62.4$ and $200 \mathrm{GeV}$ for $-3.7<\eta<-2.3$. Errors shown are systematic only and include those for $\left\langle N_{\text {part }}\right\rangle$. Results from HIJING are shown as lines (solid for $\mathrm{Au}+\mathrm{Au}$ and dashed for $\mathrm{Cu}+\mathrm{Cu}$ ). Bottom panel: Same as above, for both photons and charged particles from PHOBOS [10]. 
Figure 4 (top panel) shows the variation of photon multiplicity per average number of participating nucleon pair with $\left\langle N_{\text {part }}\right\rangle$ for $\mathrm{Au}+\mathrm{Au}$ and $\mathrm{Cu}+\mathrm{Cu}$ at 62.4 and $200 \mathrm{GeV}$ within the range $-3.7<\eta<-2.3$. We observe that within the systematic errors, the photon multiplicity scales with $\left\langle N_{\text {part }}\right\rangle$ at forward rapidities. This indicates that the photon production at forward rapidities is due to soft processes. For collisions with similar $\left\langle N_{\text {part }}\right\rangle$, the photon multiplicity is similar for $\mathrm{Au}+\mathrm{Au}$ and $\mathrm{Cu}+\mathrm{Cu}$ at a given beam energy. Also shown in the figure are results from HIJING (solid lines for $\mathrm{Au}+\mathrm{Au}$ and dashed lines for $\mathrm{Cu}+\mathrm{Cu}$ ). Considering the systematic errors shown, the HIJING results compare well with the data for most of the collision centralities studied.

Figure 4 (bottom panel) shows the comparison of photon multiplicity per average number of participating nucleon pair vs. $\left\langle N_{\text {part }}\right\rangle$ and the corresponding data for charged particles from the PHOBOS experiment for the range $-3.7<$ $\eta<-2.3$. Like photon production, the charged particle multiplicity at forward rapidities is found to scale with $\left\langle N_{\text {part }}\right\rangle$. For similar $\left\langle N_{\text {part }}\right\rangle$, the charged particle production in the region $-3.7<\eta<-2.3$ is also found to be similar for $\mathrm{Au}+\mathrm{Au}$ and $\mathrm{Cu}+\mathrm{Cu}$ at a given beam energy. The photon production per average number of participating nucleon pair is slightly lower compared to that for charged particles. A constant straight line combined fit to the charged particle results for $\mathrm{Au}+\mathrm{Au}$ and $\mathrm{Cu}+\mathrm{Cu}$ in Fig. 4 at $\sqrt{s_{\mathrm{NN}}}=200 \mathrm{GeV}$ gives 3.8 \pm 0.2 , while that for photons yields $3.2 \pm 0.1$. For $\sqrt{s_{\mathrm{NN}}}=62.4 \mathrm{GeV}$ the values are $2.2 \pm 0.1$ and $1.6 \pm 0.05$ for charged particles and photons, respectively. The difference may be due to the contribution of protons to charged particles at forward rapidity. The measurements $(-3.7<\eta<-2.3)$ are carried out close to the fragmentation region, where protons play an increasingly larger role $[13,22]$. The ratio of the number of charged particles to photons in the range $-3.7<\eta<-2.3$ is found to be $1.4 \pm 0.1$ and $1.2 \pm 0.1$ for $\sqrt{s_{\mathrm{NN}}}=$ $62.4 \mathrm{GeV}$ and $200 \mathrm{GeV}$, respectively.

\subsection{Longitudinal Scaling}

Previously it was reported that both charged particle $[10,11]$ and photon pseudorapidity density $[12,13]$, normalized by the average number of participating nucleon pairs as a function of $\eta-y_{\text {beam }}$, where $y_{\text {beam }}$ is the beam rapidity, is independent of beam energy. Further, it was observed that such longitudinal scaling was centrality dependent for charged particles, but was centrality independent for photons $[12,13]$. Figure 5 shows the photon pseudorapidity density normalized by the average number of participating nucleon pairs as a function of $\eta-y_{\text {beam }}$, for selected centralities (for the sake of clarity) for $\mathrm{Au}+\mathrm{Au}$ and $\mathrm{Cu}+\mathrm{Cu}$ at $\sqrt{s_{\mathrm{NN}}}=62.4$ and $200 \mathrm{GeV}$. The $y_{\text {beam }}$ values for 62.4 and $200 \mathrm{GeV}$ are -4.19 and -5.36 , respectively. The $\mathrm{Cu}+\mathrm{Cu}$ results are shifted by 0.1 units in $\eta$ for sake of clarity. The solid line is a second order polynomial of the form 

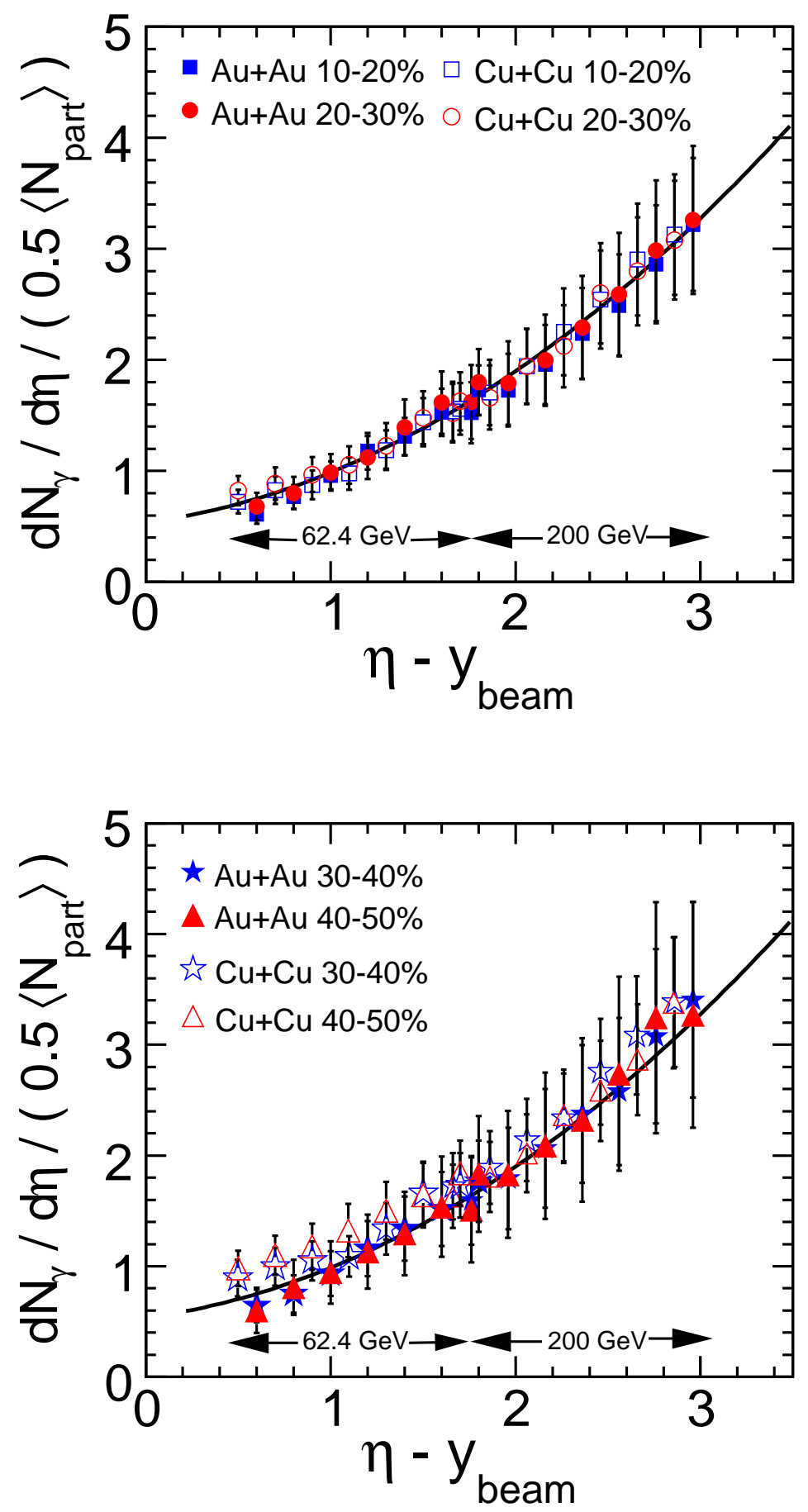

Fig. 5. (color online) Photon pseudorapidity distributions normalized by the average number of participating nucleon pairs for different collision centralities are plotted as a function of pseudorapidity shifted by the beam rapidity $(-5.36$ for $200 \mathrm{GeV}$ and -4.19 for $62.4 \mathrm{GeV}$ ) for $\mathrm{Au}+\mathrm{Au}$ and $\mathrm{Cu}+\mathrm{Cu}$ collisions at $\sqrt{s_{\mathrm{NN}}}=62.4$ and $200 \mathrm{GeV}$. Errors are systematic only, statistical errors are negligible in comparison. For clarity of presentation, results for only four centralities are shown. The $\mathrm{Cu}+\mathrm{Cu}$ data are shifted by 0.1 unit in $\eta-y_{\text {beam }}$. The solid line is a second order polynomial fit to the data (see text for details). 
$0.54+0.22\left(\eta-y_{\text {beam }}\right)+0.23\left(\eta-y_{\text {beam }}\right)^{2}$, fitted to all the data of Fig. 5 . A fit to the ratio of data to this function for the results in the upper panel yields a value of $0.96 \pm 0.01$ and those on the lower panel yields $1.03 \pm 0.01$. The results demonstrate that the longitudinal scaling for produced photons is independent of colliding ion species. In addition we re-confirm that such scaling for photons is independent of beam energy and collision centrality as reported earlier $[12,13]$.

\section{Summary}

Photon multiplicity distributions are measured at forward rapidity $(-3.7<$ $\eta<-2.3)$ for $\mathrm{Au}+\mathrm{Au}$ and $\mathrm{Cu}+\mathrm{Cu}$ collisions at $\sqrt{s_{\mathrm{NN}}}=62.4$ and $200 \mathrm{GeV}$ using the photon multiplicity detector in the STAR experiment at RHIC. As expected, the photon yield increases with decreasing $|\eta|$ (towards midrapidity), and is larger for collisions at higher energies. The photon multiplicity per participating nucleon pair is observed to be independent of collision centrality indicating that photon production is dominated by soft processes. A similar observation is made for charged particles, although their production is slightly higher. This slightly higher production of charged particles than photons could be due to the contribution of baryons to the charged particles, which may come from baryon transport and contribution from beam protons. On the other hand, photons are mainly from the decay of mesons $\left(\pi^{0}\right)$. For collisions with similar average number of participating nucleons, the photon yields are similar for $\mathrm{Au}+\mathrm{Au}$ and $\mathrm{Cu}+\mathrm{Cu}$ for a given colliding beam energy. The photon production per unit rapidity per average number of participating nucleon pair vs. $\eta-y_{\text {beam }}$ shows longitudinal scaling which is independent of beam energy, collision centrality and colliding ion species.

We thank the RHIC Operations Group and RCF at BNL, the NERSC Center at LBNL and the Open Science Grid consortium for providing resources and support. This work was supported in part by the Offices of NP and HEP within the U.S. DOE Office of Science, the U.S. NSF, the Sloan Foundation, the DFG cluster of excellence 'Origin and Structure of the Universe', CNRS/IN2P3, STFC and EPSRC of the United Kingdom, FAPESP CNPq of Brazil, Ministry of Ed. and Sci. of the Russian Federation, NNSFC, CAS, MoST, and MoE

of China, GA and MSMT of the Czech Republic, FOM and NWO of the Netherlands, DAE, DST, and CSIR of India, Polish Ministry of Sci. and Higher Ed., Korea Research Foundation, Ministry of Sci., Ed. and Sports of the Rep. Of Croatia, Russian Ministry of Sci. and Tech, and RosAtom of Russia. 


\section{References}

[1] BRAHMS Collaboration, I. Arsene et al., Nucl. Phys. A 757 (2005) 1; PHOBOS Collaboration, B. B. Back et al., Nucl. Phys. A 757 (2005) 28; STAR Collaboration, J. Adams et al., Nucl. Phys. A 757 (2005) 102; PHENIX Collaboration, K. Adcox et al., Nucl. Phys. A 757 (2005) 184.

[2] H. Heiselberg, Phys. Rep. 351 (2001) 161; WA98 Collaboration, M. M. Aggarwal et al., Phys. Rev. C 65 (2002) 054912.

[3] B. Mohanty and J. Serreau, Phys. Rep. 414 (2005) 263; WA98 Collaboration, M. M. Aggarwal et al., Phys. Rev. C 64 (2001) 011901(R).

[4] PHENIX Collaboration, K. Adcox et al., Phys. Rev. Lett. 86 (2001) 3500.

[5] L. V. Gribov, E. M. Levin and M. G. Ryskin, Phys. Rep. 100 (1983) 1; J. P. Blaizot and A. H. Mueller, Nucl. Phys. B 289 (1987) 847.

[6] L. McLerran and R. Venugopalan, Phys. Rev. D 49 (1994) 2233; L. McLerran and R. Venugopalan, Phys. Rev. D 50 (1994) 2225; Y. V. Kovchegov, Phys. Rev. D 54 (1996) 5463; E. Iancu and L. McLerran, Phys. Lett. B 510 (2001) 145; A. Krasnitz and R. Venugopalan, Phys. Rev. Lett. 84 (2000) 4309.

[7] J. D. Bjorken, Phys. Rev. D 27 (1983) 140; L. D. Landau, Izv. Akad. Nauk Ser. Fiz. 17 (1953) 51; S. Belenkij and L. D. Landau, Usp. Fiz. Nauk. 56 (1955) 309; Nuovo Cim. Suppl. 3S10 (1956) 15; R. C. Hwa and K. Kajantie, Phys. Rev. D 32 (1985) 1109; J. Alam et al., Annals of Phys. 286 (2000) 159; B. Mohanty and J. Alam, Phys. Rev. C 68 (2003) 064903.

[8] P. K. Netrakanti and B. Mohanty, Phys. Rev. C 71 (2005) 047901; J. Aichelin and K. Werner, Phys. Lett. B 300 (1993) 158.

[9] J. Benecke et al., Phys. Rev. 188 (1969) 2159.

[10] PHOBOS Collaboration, B.B. Back et al., Phys. Rev. Lett. 87 (2001) 102303. PHOBOS Collaboration, B. B. Back et al., Phys. Rev. Lett. 91 (2003) 052303.

[11] BRAHMS Collaboration, I. G. Bearden et al., Phys. Lett. B 523 (2001) 227; BRAHMS Collaboration, I. G. Bearden et al., Phys. Rev. Lett. 88 (2002) 202301.

[12] STAR Collaboration, J. Adams et al., Phys. Rev. Lett. 95 (2005) 062301; P. K. Netrakanti, Ph. D. Thesis, Jadavpur University (2008); M. Sharma, Ph. D. Thesis, Panjab University (2008); N. Gupta, Ph. D. Thesis, Jammu University (2008); D. Das, Ph. D. Thesis, Jadavpur University (2008); and S. M. Dogra, Ph. D. Thesis, Jammu University (2009). http://drupal.star.bnl.gov/STAR/theses.

[13] STAR Collaboration, J. Adams et al., Phys. Rev. C 73 (2006) 034906.

[14] K. H. Ackermann et al., Nucl. Instr. Meth. A 499 (2003) 624.

[15] X-N. Wang and M. Gyulassy, Phys. Rev. D 44 (1991) 3501. 
[16] B. Zhang, C. M. Ko, B. A. Li, and Z. Lin, Phys. Rev. C 61 (2000) 067901.

[17] M. M. Aggarwal et al., Nucl. Instr. Meth. A 499 (2003) 751; M. M. Aggarwal et al., Nucl. Instr. Meth. A 488 (2002) 131.

[18] F. S. Bieser et al., Nucl. Instr. Meth. A 499 (2003) 766.

[19] STAR Collaboration, B. I. Abelev et al., Phys. Rev. C 79 (2009) 034909.

[20] WA98 Collaboration, M. M. Aggarwal et al., Phys. Lett. B 458 (1999) 422.

[21] V. Fine and P. Nevski, in Proceedings of CHEP-2000, Padova, Italy.

[22] BRAHMS Collaboration, I. G. Bearden et al., Phys. Rev. Lett. 93 (2004) 102301; BRAHMS Collaboration, I. C. Arsene et al., arXiv: 0901.0872v1 [nuclex]. 